\title{
CONF-951276-1
}

\section{AN IMPROVED RESPONSE SURFACE METHODOLOGY ALGORITHM WITH AN APPLICATION TO TRAFFIC SIGNAL OPTIMIZATION FOR URBAN NETWORKS}

\author{
Shirish S. Joshi \\ Ajay K. Rathi
}

Oak Ridge National Laboratory

Center for Transportation Analysis

P.O. Box 2008, MS 6206

Oak Ridge, TN 37831 , U.S.A.

\author{
Jeffrey D. Tew \\ ISQ Department \\ P.O. Box 6696 \\ Consolidate Freightways, Inc. \\ Portland, OR 97208, U.S.A.
}

\begin{abstract}
This paper illustrates the use of the simulationoptimization technique of response surface methodology (RSM) in traffic signal optimization of urban networks. It also quantifies the gains of using the common random number (CRN) variance reduction strategy in such an optimization procedure. An enhanced RSM algorithm which employs conjugate gradient search techniques and successive second-order models is presented instead of the conventional approach. An illustrative example using an urban traffic network exhibits the superiority of using the CRN strategy over direct simulation in performing traffic signal optimization. The relative performance of the two strategies is quantified with computational results using the total network-wide delay as the measure of effectiveness (MOE).
\end{abstract}

\section{INTRODUCTION}

In many simulation studies the analyst is interested in exploring the settings of the non random inputs, called the factors, to yield an optimal output, called the response. For this purpose, simulation runs are performed at different factor levels, to investigate the effects of the factors on the response. The analyst then attempts to estimate a metamodel of a selected response, that is. a linear or non-linear model of the mean response in terms of the factors. This estimated metamodel is used to perform a sequential search in an attempt to find the optimal (or near optimal) value for the response. This process of achieving optimality of responses will be referred to as the simulationoptimization technique. The sequential search emploved by such techniques depend extensively on the estimation of the metamodel parameter. The quality of such a search depends upon the qualitv of estimation of these parameters. Since this procedure assumes stochastic nature of responses, the estimates of the unknown parameters of such metamodels inherit certain amount of variability. The quality of these estimates is characterized by their variance. Reduced variance of these estimates suggests improved search directions for the optimization procedure.

Variance reduction techniques (VRT) try to reduce the variance of the estimates of interest. Among the various correlation-induction techniques used as VRT, such as the common random number strategy, antithetic variates, control variates, etc., the focus of this paper is on the use of the common random number strategy.

The goal of this paper is to demonstrate the use of common random number (CRN) strategy in the simulation-optimization technique of response surface methodology (RSM). After providing a theoretical framework illustrating the superiority of the using common random numbers over direct simulation in RSM studies, this idea is demonstrated with illustrative examples on a sample urban network. Quantitative gains of using the CRN strategy over ordinary simulation, or the Independent Streams strategy (referred to, as the IS strategy), are also illustrated.

The remainder of this section discusses a modified RSM algorithm and the CRN strategy. Section 2 develops the theoretical framework, illustrative exampies are included in Section 3, and Section 4 presents the conclusions of this study.

\subsection{RSM}

This sub-section presents a standard RSM algorithm offered by Myers (1976). For the modified RSM algorithm used by this work, the reader is referred to Joshi, Sheraii, and Tew (1994) .

Step 1: Fit a first-order regression model to the mean

"The submitted manuscript has been authored by a contractor of the U.S. Government under contract No. DE-AC05-960R22464. Accordingly, the U.S. Government retains a nonexclusive, royalty-free license to publish or reproduce the published form of this contribution, or allow others to do so, for U.S Government purposes." 
response over some restricted region of the factor space. Step 2: Estimate the path of steepest descent using the first-order model.

Step 3: Search along the path of steepest descent until no further improvement in the mean response is observed.

Step 4: Estimate the most favorable response based on the first three steps.

Step 5: Repeat Steps I through 4 over a new region. If curvarure is evident, then perform an elaborate search using a second-order experimental design. Report the optimum solution.

For Step 1, the experimenter should judicioutly select an experimental design which has desirable properties such as minimum variance in order to obtain berter estimates of the unknown parameters. The firstorder linear model is represented as

$$
y_{i}=\beta_{0}+\sum_{i=1}^{k} \beta_{x_{i}}+\epsilon_{y},
$$

where $y_{7}$ is the response at the $j$ th replication and the ith design point. $x_{1}, x_{3} \ldots, x_{2}$ are the $k$ factor variables, $\beta_{1}$ are the unknown parameters of the linear model, and $e_{i i}$ is the error term at the ith design point and the $j$ th replication $(i=1,2, \ldots, m, \mathrm{j}=1,2, \ldots, r, l=1,2, \ldots, k)$.

Let $b$, be the estimates of $\beta,(l=1,2, \ldots, k)$. Step 2 uses these estimates to compute the path of steepest descent given by $\left(-b_{1},-b_{3}, \ldots,-b_{1}\right)$. Responses are observed along this path until no further improvement is achieved. At this point, if curvature is evident, then a second-order model is used and is given by

$$
y_{i l}=\beta_{0}+\sum_{i=1}^{k} \beta_{z_{i}}+\sum_{h=1} \beta_{h} x_{k} x_{i}+\sum_{i=1}^{k} \beta_{i} x_{j}^{2}+\epsilon_{j},
$$

where $\beta_{b} \beta_{h b}$ ard $\beta_{b}$ are the unknown parameters of the second-order model $(l=1,2, \ldots, k, h>h)$ and the remaining terms are as defined in (1). This model is used to perform a canonical analysis which involves evaluation and analysis of the stationary point. Using this analysis, an optimum is reported, perhaps following some additional investigation in the case of a detected ridge system. The tist sub-section presents the CRN strategy which is combined with this algorithm to improve the RSM technique in practice.

\subsection{Common Random Number Strategy}

The idea of the CRN strategy is to compare altemative simulation models under similar experimental conditions in order to improve confidence that observed differences in performance are due to the differences in the model structure rather than to the differences in the experiment itself (see p. 61 of Law and Kelton 1991). Under the CRN strategy, the same set of random number strtams, $\boldsymbol{R}_{i}=\left(r_{i,}, r_{i 3}, \ldots, r_{t g}\right)$ is applied to all $m$ design points in the theplicate where $g$ is the number of streams used to drive the simulation model. Also, independent random number streams are used across replicates of the experimental design. Replications reduce the variance of the outputs and also present means of computing pure error.

For the CRN strategy applied to simulation experiments, we make the following assumptions:

1. The response variance is constant across all design points, so that for $j=1,2, \ldots, m$ and $i=1,2, \ldots, r$,

$$
\mathbf{o}_{j}^{2}=\operatorname{var}\left(y_{j}\left(\boldsymbol{R}_{j}\right)\right)=\mathrm{o}^{2} \text {. }
$$

2. There is a constant nonnegative correlation between all pairs of responses within a given replicate. $y_{i j}$ and $y_{i k}$ $(j \neq k)$. That is, for $j \neq k$, and $l<j, k\left\langle m\right.$, and $0<p_{+}<l$,

$$
\operatorname{corr}\left(y_{i p} y_{w}\right)=p
$$

3. The vector of responses comprising the th replicate has a multivariate normal distribution. Under the first two assumptions, the covariance matrix between observations within a replicate is given by

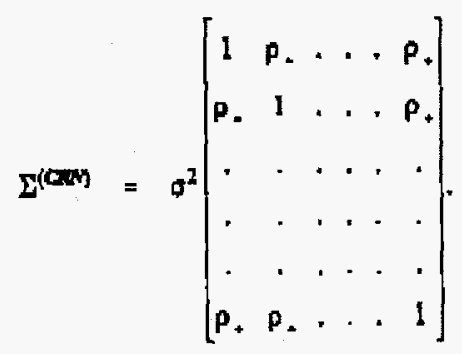

Note that the variance on the estimates of the unknown perameters of the linear model in (l) is $\sigma^{2}$ under IS strategy, but is reduced by a factor of $\left(1-p_{+}\right) a^{2}$ under the CRN strategy (see Joshi and Tew 1995). The next section discusses the gains of using the CRN over IS strategy in RSM studies.

\section{ANALYTICAL RESULTS FOR RSM UNDER THE CRN STRATEGY}

As the gradient search is performed, the statistic of interest is the difference berween the responges along the path. If we denote $\bar{y}_{0}$ ts the mean response at the center of the current design, and $\vec{y}, j=1,2, \ldots, w)$ as the $w$ mean responses along the gradient search path, then our 
statistics of interest are $\bar{y}_{j} \bar{y}_{j-1}(j=1,2, \ldots, w)$. Under the CRN scheme, we apply the same random number strearns to drive the stochastic components to obtain simulation responses $\bar{y}_{j}(j=0,1, \ldots, w)$. By the first two assumptions of the CRN strategy, we have positive correlations $p_{+}$induced among all these responses. Under the independent strategy, $(j=1,2, \ldots, w)$

$$
\operatorname{var}\left(y_{J}-y_{j-2}\right)=\frac{2 \sigma^{2}}{r}
$$

while under the CRN strategy,

$$
\operatorname{var}\left(y_{j}-y_{j-1}\right)=\frac{2 a^{2}\left(1-\rho^{2}\right)}{r}
$$

where $r$ is the number of replications performed at each design point, and $\sigma^{2}$ is the homogeneous variance of the response at each design point (see Joshi and Tew 1995):

From (7) we see that as $p \rightarrow 1$. the variance on the statistic of interest in the pradient search procedure approaches zero. That is, if we can induce large covariances or correlations between responses, then we have a larger reduction in variance of the statistics of interest under the gradient search method. The expected reduction in variance being the magnitude of the induced correlation.

The advantage of using CRN for the gradient search can be characterized in another way. In the gradient search, assume that once the step length $\lambda$ is fixed. we need to take exactly $w$ steps before the response starts increasing. That is. after fixing the step length, we should get responses to improve exactly until the $(w-1)$ st step, and on the w-th step, the response should increase forcing us to stop the gradient search procedure. However, due to natural variation in $y$, the variance in the mean responses may be so large that this could result in the search taking something other than exactly w steps along the gradient. We would like to increase the probability that the experimenter stops after exactly $w$ steps.

In other words, the gradient search procedure is similar to performing sequential tests of the form $j=$ $1,2, \ldots, w)$

$$
\mathrm{H}_{0}: \bar{y}_{j}-\bar{y}_{j-1} \leq 0, \mathrm{vs}, \mathrm{H}_{1}: \bar{y}_{j}-\bar{y}_{j-1}>0 \text {. }
$$

If the search is performed accurately, then the experimenter should fail to reject the first $w$-l tests, and reject the $w$-th test. For this purpose, let us first define $d_{i}=\bar{y}_{i=1}-\bar{y}_{1},(i=1,2, \ldots, w-1)$, and $d_{v}=\bar{y}_{k}-\bar{y}_{v-1}$. Maximizing the probability that the experimenter stops after exactly $w$ steps, is therefore equivalent to maximizing the following probability

$$
\operatorname{Pr}\left(d_{1} \geq 0, d_{2} \geq 0, \ldots, d_{4} \geq 0\right)
$$

This probability is different under the IS and CRN strategies. We now prove that the above probability is higher under the CRN straregy than under the is strategy. Proving this would also indicate that the power of the test under the CRN straregy is greater than that under the IS strategy for the following hypothesis test

$\left.H_{0}: d_{1}\right) 0, d_{2}, 0, \ldots, d_{w}>0$, versus,

$\mathrm{H}_{1}$ : Any of the above conditions is violated.

We know that the $d$,'s are not independent and so the tests on the $d_{i}^{\prime}$ s are also not independent. Now,

$$
\operatorname{var}\left(d_{1}\right)=\operatorname{var}\left(\bar{y}_{1}\right)+\operatorname{var}\left(\bar{y}_{i-1}\right)-2 \operatorname{cov}\left(\bar{y}_{i} \bar{y}_{1-1}\right)
$$

Note that while $\operatorname{cov}\left(\bar{y}_{n} \bar{y}_{t-1}\right)=0$ under the IS strategy, we induce covariances between pairs of responses under he CRN strategy such that $\operatorname{cov}\left(\bar{y}_{i}, \bar{y}_{i=1}\right)=\rho^{2} a^{2}$. Thus, we ger for the IS strategy,

$$
\operatorname{var}(d)=\frac{2 \sigma^{2}}{y},
$$

and for the CRN strategy,

$$
\operatorname{var}(d)=\frac{2 \sigma^{2}(1-p)}{r} .
$$

Also, under the IS strategy

$$
\operatorname{cov}\left(d_{r} d_{t-1}\right)=\operatorname{cov}\left(\left(\bar{y}_{i}-\bar{y}_{i-1}\right), \bar{y}_{t-1}-\bar{y}_{p}\right)=\frac{-\sigma^{2}}{r},
$$

and under the CRN strategy,

$$
\operatorname{cov}\left(d_{p} d_{i-1}\right)=\operatorname{cov}\left(\bar{y}_{1}-\bar{y}_{i-1}\right)\left(\bar{y}_{i+1}-\bar{y}_{p}\right)=\frac{\sigma^{2}\left(1-\mathrm{p}^{2}\right)}{r} .
$$

The variance-covariance matrix of $d_{i} s$ is thus a tridiagonal matrix denoted by $\theta$. Note that the structure of this marrix is identical under the two simulation strategies, and this matrix is represented as 


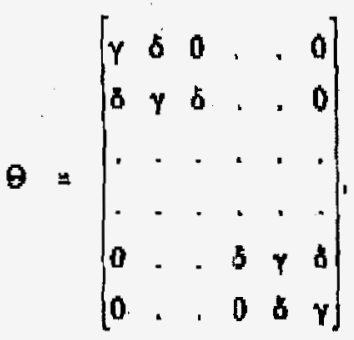

where $\gamma=20^{2} / r$ under the IS strategy and $\gamma=20^{*}$ $\left(1-\rho^{2}\right) / r$ undet the CRN strategy. Also, $\delta=-\sigma^{2} / r$ under the IS strategy and $\delta=-\sigma^{2}\left(1-p^{2}\right) / r$ under the CRN strategy.

To obtain independent tests on $d / s$, we use an orthogonal transformation denoted by $\Gamma$, such that

$$
\theta^{+}=\Gamma \theta \Gamma^{\prime}
$$

is a diagonal marrix. Nole that $\Gamma$ is an orthogonal matrix whose columns comprise of the eigenvectors of $\theta$. The transformation $\Gamma$ will be different under IS and CRN strategies, but will have the same matrix structure. Thus, if we transform $d^{\prime} \equiv\left(d_{1}, d_{2}, \ldots, d_{1}\right)$ to $d^{2}$ such that

$$
d^{*}=\Gamma \cdot d
$$

and let $d^{\prime}=\left(d_{2}, d_{2}, \ldots, d_{q}\right)$, then the tests performed ot $d_{i}^{*}$ are independent The goal then becomes to maximize the following probability

$$
\left.P r\left(d_{1}^{*}\right) 0, \ldots, d_{-}^{*}>0\right)=\operatorname{Pr}\left(d_{1}^{*}>0\right) \cdots P r\left(d_{-}^{*}>0\right)
$$

Using $\phi$ as the standard normal cumutative distribution function, we can standardize the individual $d_{1}^{\prime} s$ under both strategies. For notational convenience, $d_{i}^{-1} s$ are replaced by $d_{f}^{\prime} s$ so that the $a_{j}^{\prime} s$ under the CRN strategy would actually refer to the $d_{i}$ 's from (16). The probabilities are then obtained as $(i=1,2, \ldots w)$,

$$
\left.P r\left(d_{1}\right) 0\right)=\phi\left(\frac{\bar{d}_{i}}{\sigma_{d} w}\right)
$$

where $\vec{d}_{i}$ is the mean of $d,(i=1,2, \ldots, w)$ and $\sigma_{d}$ is the homogeneous variance of all $d_{j}$. Hence the homogeneous variance of all $\bar{d} ' s$ is $o_{d}^{2} / w$. The goal in (18) is now equivalent to minimizing $o_{\mu}$. Equations (10) and (11) give the expressions for $\sigma^{2}{ }_{d}$ under the IS and CRN strategy, respectively. We notice that under the CRN strategy, $\sigma_{d}$ is less than that under IS strategy by a factor of $\sqrt{1-\rho^{2}}$, thus showing the superiority of the CRN over the IS strategy.

\section{COMPUTATIONAL RESULTS}

An urban traffic network shown in Figure 1 is used to compare the relative effectiveness of $\mathrm{CRN}$ and IS surategies in the context of the RSM algorithm. The simulation model used for the purpose of this study is a mícroscopic model developed by the Federal Highway Administration (FHWA), called TRAF-NETSIM. To justify the validity of these quantitative results, five optimization searches were conducted using the same initial solution but employing different random number seeds under each search. The relative performance of the two strategies is quantified using the total delay in the network as the measure of effectiveness (MOE).

Table 1 represents the geometric characteristics of the urban trafiic network under sudy. It is assumed that at any stage of this search, the model can be represented as a first-order model in $x_{1}$ and $x_{2}$ as in (1) or a secondorder model as given by (2). A $2^{2}$ factorial experiment is used to construct the first-order model and a central composice design for the second-order model. Two replications are performed at each design point. The decision variables are the green Splits for traffic at node 5 for approach node $13\left(x_{3}\right)$ and at node 8 for approach node $14\left(x_{2}\right)$. The starting solution was 32 seconds for each variable. Five optimization searches are conducted under both sirategies.

Table 2 exhibits the optimal solution for the total delay in the network in person-minutes reported under both strategies. The average delay in the network across all five searches is 15085 under the CRN strategy and 15597 under the IS strategy. The RSM procedure carried out under the CRN strategy yields a delay estimate which is 8 hours less than that carried out under the IS strategy, thus illustrating the superiority of the CRN straregy. We also observed that only one of the five searches conducted under the IS strategy achieves improving direction, in contrast to all five searches conducted under the CRN strategy.

Table 3 indicates the average gain in delay per simulation run. This can be en extemely important scaristic for practitioners, especially if the nerwork under study is large and therefore requires large amounts of simulation time. In such a situation, the analyst would like to achieve the optimal (or near optimal) solution in as small number of simulation runs as possible. In addition, it will also necessitate a reduction in the number of replications at each design point.

\section{CONCLUSIONS}

The application of the CRN strategy in a standard simulation-RSM algorithm is demonstrated with an illustrative maffic network. Correlation-induction 
strategies have not been employed in sequential simulation-RSM studies in taffic optimization and hence this application of the CRN strategy is an addition to the existing literature. Al each stage of the RSM algorithm, analytical results to show the gains of the CRN stritery over dintet simulation are developed. To quantify the gains of the CRN surategy over the is strategy, an cmpirical investigation on the example was described in Stetion 3. These performance measures exemplify the superiority of the CRN strategy over direct simulation.

Table I: Network Description

\begin{tabular}{|c|c|}
\hline $\begin{array}{l}\text { Link length } \\
\text { All links }\end{array}$ & $500 \mathrm{ft}$. \\
\hline $\begin{array}{l}\text { Signal Control } \\
\text { Nodes } 5,6,7 \text {, and } 8 \\
\text { All other nodes }\end{array}$ & $\begin{array}{l}\text { Signal Control. } \\
\text { Perpetual Green. }\end{array}$ \\
\hline $\begin{array}{l}\text { Number of Lanes } \\
\text { All links }\end{array}$ & Two lanes. \\
\hline $\begin{array}{l}\text { Entry Voluome } \\
\text { All nodes }\end{array}$ & $1600 \mathrm{vph}$. \\
\hline $\begin{array}{l}\text { Tuming Monement } \\
\text { At four-way } \\
\text { thteruections } \\
\text { At other } \\
\text { intersection. }\end{array}$ & $\begin{array}{l}25 \% \text { lett, } 50 \% \text { through, } \\
25 \% \text { right } \\
100 \% \text { through. }\end{array}$ \\
\hline
\end{tabular}

Table 2: Total Delay in the Nerwork

\begin{tabular}{|c|c|c|}
\hline \multirow{2}{*}{ Search \# } & \multicolumn{2}{|c|}{$\begin{array}{c}\text { Optimal reported total } \\
\text { delay in the netwotk } \\
\text { (person minutes) }\end{array}$} \\
\cline { 2 - 3 } & $\begin{array}{c}\text { CRN } \\
\text { Strategy }\end{array}$ & Is Strategy \\
\hline 1 & 15038 & $15794^{\circ}$ \\
\hline 2 & 15087 & $15616^{\circ}$ \\
\hline 3 & 15113 & $15690^{\circ}$ \\
\hline 4 & 15054 & 15203 \\
\hline 5 & 15135 & $15681^{*}$ \\
\hline Average & 15085 & 15597 \\
\hline
\end{tabular}

* indicates that the search failed.

Table 3: Improvemeat per simulation run

\begin{tabular}{|c|c|c|}
\hline & CRN Strategy & $\begin{array}{c}\text { IS } \\
\text { Strategy }\end{array}$ \\
\hline $\begin{array}{c}\text { Reduced delay } \\
\text { (persol minutes) } \\
\text { per simulation } \\
\text { run }\end{array}$ & 19.45 & 8.43 \\
\hline
\end{tabular}

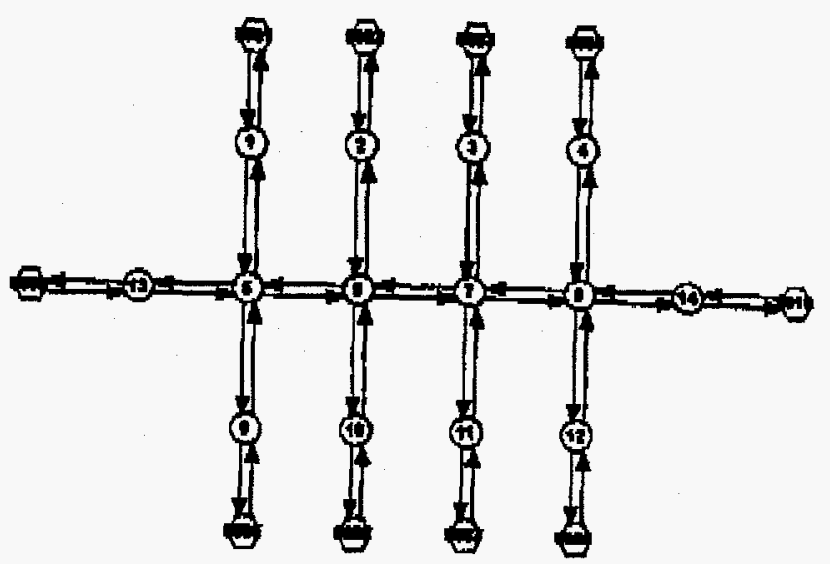

Figure 1: Traffic Network 


\section{ACKNOWLEDGMENTS}

he authors thank the editors for their comments. The uthors also wish to convey their sincere appreciation to research assistant, Kathy Dyke for her helpful suggestions.

\section{REFERENCES}

Federal Highway Administration. 1980. Traffic Network Analysis with NETSIM - A User Guide. Federal Highway Administration, U.S. Department of Transportation.

Joshi S.S., H.D. Sherali, and J.D. Tew. 1994. An Enhanced RSM Algorithm Using Gradient-Deflection and Second-Order Search Strategies. In Proceedings of the 1994 Winter Simulation Conference, ed. J. Tew, S. Manivannan, D. Sadowski, and A. Seila: 297-304. Instinte of Electrical and Electronics Engineers, Orlando, Florida.

Joshi, S.S., and J. D. Tew. 1995. Statistical Analysis and Validation Procedures Under the Common Random Number Correlation Induction Strategy for Multipopulation Simulation Experiments. European Journal of Operational Research, to appear.

Law, A. M. and W. D. Kelton. 1991. Simulation Modeling and Analysis, 2d ed. New York: McGraw-Hill. Myers, R. H. 1976. Responie Surface Methodology. Ann Arbor, MI: Edwards Brothers, Inc.

\section{AUTHOR BIOGRAPHIES}

SHIRISH S. JOSHI is a post doctoral research associate at the Oak Ridge National Laboratory in the Intelligent Transportation Systems (ITS) research group. He received his B.S. in Electrical Engineering from the Bombay Universiry in 1986, M.S. in Mathematical Sciences from Virginia Commonwealth Universiry in 1989, and M.S. and Ph.D. in Industrial and Systems Engineering from Vilginia Polytechnic Institute and State University in 1991 and 1993 respectively. His current research interests include output analysis of traffic simulations. simulation-optimization techniques, variance reduction techniques, and design of experiments.

AJAY K, RATHI is the R\&D Program Manager for the Intelligent Transportation Systems (ITS) Research Group at the Oak Ridge National Laboratory. Dr. Rathi has over I2 years of research and Project Management Experience in transportation systems modeling and analysis. He holds an M.S. and a Ph.D. in Civil Engineering from the Ohio State University. $\mathrm{He}$ is a registered professional engineer and a member of several committees of the Transportation Research Board, the ITS America steering committee on Advanced Traffic Management Systems, the American Society of Civil Engineers, and the Society for Computer Simulation. He is the author of nearly 100 technical books, reports, papers, and publications.

JEFFREY D. TEW is a Senior Systems Engineer at Consolidated Freightways, Ine, and an Adjunct Associate Professor at the Oregon Graduate Institute. Prior to these appoinments he was on the faculty in the Industrial and Systems Engineering Department at the Virginia Polytechnic Institute and State Universiry. He has published numerous articles in many archival journals such as EJOR, JSCS, Management Sciente, Operations Research, and SCS Transactions. He was the 1994 WSC Proceedings Editor as well as the Vice President/Fresident elect of the TIMS College on Simulation. He received a B.S. in Mathematics in 1979. an M.S. in Statistics in 1981. and a Ph.D. in Industrial Engineering in 1986, all from Purdue University. His cutrent research interests include variance reduction techniques, simulation optimization, and the design of experiments. He is a member of Alpha Pi Mu, ACM, ASA, IME, INFORMS, SCS. and Sigma Xi. 